\title{
Fine needle aspiration cytology findings of mucinous carcinoma of breast: a study of eight cases with histological correlation
}

\author{
Adhikari RC ${ }^{1}$, Jha $\mathrm{A}^{1}$, Sayami $\mathrm{G}^{1}$ \\ ${ }^{I}$ Department of Pathology, Tribhuvan University Teaching Hospital, Kathmandu, Nepal
}

\section{Keywords: \\ Breast; \\ Fine needle aspiration cytology; \\ Mucinous carcinoma}

\begin{abstract}
Background: Pure mucinous carcinoma is a rare histologic type of mammary neoplasm and may exist as mixed mucinous-ductal carcinoma. This study aims to describe fine needle aspiration cytology features of mucinous carcinoma of breast diagnosed in Tribhuvan University Teaching Hospital, Nepal over the period of 2 years and to correlate with histological findings.

Materials and Methods: There were a total of 8 cases of mucinous carcinoma of breast, diagnosed on fine needle aspiration cytology in TUTH during the period from April 2010 to March 2012. The patient's age, laterality of involvement, fine needle aspiration cytology features, gross and histological findings, type of surgery performed and tumor size were evaluated. Fine needle aspiration cytology smears were evaluated for cellularity, amount of mucin, chicken-wire vasculature and nuclear atypia.

Results: Fine needle aspiration cytology provided a pre-operative diagnosis of mucinous carcinoma in 5 cases and mixed mucinous carcinoma-invasive ductal carcinoma in 3 cases. Histological examination showed pure mucinous carcinoma in 3 cases, mixed mucinous carcinoma-invasive ductal carcinoma in 4 cases and mixed cribriform carcinoma and ductal carcinoma in 1 case.

Conclusion: Mucinous carcinoma may appear clinically and radiologically benign and fine needle aspiration cytology plays important role in the correct pre-operative diagnosis. Significant nuclear pleomorphism and necrosis, in addition to extracellular mucin suggests mixed mucinous carcinomainvasive ductal carcinoma.
\end{abstract}

\section{INTRODUCTION}

Mucin-producing carcinomas of the breast have been described in pure form and as mixed mucinous-ductal type. ${ }^{1}$ Pure mucinous carcinoma (MC) is a rare entity and represent about $2 \%$ to $5 \%$ of breast cancers. ${ }^{2,3} \mathrm{MC}$ usually

\section{Correspondence:}

Dr. Ram Chandra Adhikari, MD

Consultant Pathologist, Department of pathology, Om Hospital and Research Centre, Kathmandu, Nepal

Email: rcadhikari@hotmail.com occurs in elderly female median age being older than 5560 years. ${ }^{4-6}$ This tumor shows characteristic features of mucin production by the tumor ${ }^{7}$ and in general mucinous component is $50 \%$ or more. ${ }^{8,9}$ In the mixed type tumor, the mucinous component is admixed with infiltrating epithelial component. Pure mucinous carcinoma of the breast has been reported to have a more favorable prognosis. ${ }^{10}$

Fine needle aspiration cytology (FNAC) of mucinous carcinoma shows abundant extracellular mucin, three 
Table 1: A comparison of FNAC diagnosis with histopathological diagnosis

\begin{tabular}{|c|c|c|c|c|c|}
\hline Case \# & FNAC diagnosis & Histopathological diagnosis & Grade & Tumor size & TN stage \\
\hline 1 & Mixed mucinous-ductal carcinoma & Mixed cribriform carcinoma \& invasive ductal carcinoma & 2 & $3 \times 3 \mathrm{~cm}$ & $\mathrm{~T} 2 \mathrm{~N} 2 \mathrm{a}$ \\
\hline 2 & Mucinous carcinoma & Mixed MC-IDC & 2 & $2.5 \times 2 \mathrm{~cm}$ & $\mathrm{~T} 2 \mathrm{~N} 2 \mathrm{a}$ \\
\hline 3 & Mucinous carcinoma & Mucinous carcinoma & 2 & $6 \times 5 \mathrm{~cm}$ & T3N0 \\
\hline 4 & Mixed mucinous-ductal carcinoma & Mixed MC-IDC & 2 & $4 \times 2.5 \mathrm{~cm}$ & $\mathrm{~T} 2 \mathrm{~N} 0$ \\
\hline 5 & Mucinous carcinoma & Mixed MC-IDC & 1 & $3.5 \times 2.5 \mathrm{~cm}$ & T2N0 \\
\hline 6 & Mixed mucinous-ductal carcinoma & Mixed MC-IDC & 3 & $5 \times 4 \mathrm{~cm}$ & T2N1a \\
\hline 7 & Mucinous carcinoma & Mucinous carcinoma & 1 & $4 \times 4 \mathrm{~cm}$ & T2N1a \\
\hline 8 & Mucinous carcinoma & Mucinous carcinoma & 1 & $7 \times 5 \mathrm{~cm}$ & T3N1a \\
\hline
\end{tabular}

dimensional cell groups with smooth borders, uniform cells, eccentrically located nuclei of small size and few nucleoli. ${ }^{2,3,11}$ The mucinous carcinoma should be distinguished from other mucinous lesions like mucocele like lesions, mucinous cyst and mucinous spherulosis. The problem of distinguishing pure $\mathrm{MC}$ from mixed mucinous carcinoma and invasive ductal carcinoma, NOS type (MCIDC) in fine needle aspiration (FNA) smears has been described. ${ }^{3}$

This study describes the spectrum of cytological features observed in our cases and we compared FNA smears and correspondence histological findings.

\section{MATERIALS AND METHODS}

This was a retrospective study of FNAC of breast during the period from April 2010 to March 2012 with a diagnosis of mucinous carcinoma (pure or mixed) from the record of department of pathology Tribhuvan University Teaching Hospital, Kathmandu, Nepal.

Fine needle aspiration was performed using 23 gauze needle attached to a $10 \mathrm{ml}$ syringe and aspirated material were deposited onto clean glass slides, smeared, air dried, fixed in 100\% methanol for Giemsa stain. For Papanicolaou stain, smears were wet fixed in 100\% isopropyl alcohol. Histological specimens either lumpectomy or mastectomy were fixed in formalin and processed routinely for Hematoxylin and Eosin (HE) stain.

FNAC smears were evaluated for smear cellularity, amount of mucin, chicken-wire vasculature and nuclear atypia. FNAC diagnoses were compared with histological diagnosis.

\section{RESULTS}

All patients were female and their ages ranged from 40 years to 80 years with a mean of 60 years. The mean age of patients with pure mucinous carcinomas was 63 years. All patients complained of a breast lump with a duration varying from 2 weeks to 3 years.
On examination, all patients had palpable lumps. In 3 cases, masses were mobile and appeared clinically benign. In other 3 cases, breast lumps were fixed with well defined borders and remaining 2 cases had large tumors that occupied nearly half of the breast.

Mammography and ultrasonography were done and 3 cases showed lobulated mass with well defined margins and diagnosed as benign. In remaining 5 cases, the diagnosis of malignancy was made due to presence of irregular or spiculated margin.

FNAC provided a pre-operative diagnosis of breast carcinoma in all cases. The diagnosis of mucinous carcinoma was made in 5 cases and mixed MC-IDC in 3 cases.

Cytological findings: In 5 cases, there were abundant extracellular mucins with cohesive balls, clusters, sheets and occasional single cells floating in lakes of mucin (fig. 1). The mucin was deep blue to pink material with Giemsa stain. In addition, there were numerous thin walled capillaries with chicken-wire appearance (fig. 2). Tumor cells were oval and have moderate amount of cytoplasm and eccentric hyperchromatic nucleus. Nuclear pleomorphism is mild and nucleoli are mostly inconspicuous. In addition to extracellular mucin, there were cellular smears comprising of cohesive clusters of pleomorphic epithelial cells showing vesicular chromatin and prominent nucleoli in remaining three cases. So, the diagnosis of mixed MC-IDC was made.

\section{Histopathological findings}

Gross features: Modified radical mastectomy with axillary dissection was performed in 7 cases and lumpectomy with axillary dissection in 1 case. The tumor size varied from 2.5 to $7 \mathrm{~cm}$ in greatest dimension. The masses were well circumscribed or infiltrative and cut surface showed currant-jelly like material in five cases. Two cases did not show significant gelatinous material grossly.

Microscopic features: Histological examination showed pure mucinous carcinoma in 3 cases, mixed MC-IDC in 4 cases and mixed cribriform carcinoma and ductal carcinoma in 1 case. Pure mucinous carcinomas were composed 
of extracellular mucin, in which groups of tumor cells appeared to be floating (fig. 3). Two of these were grade 1 and one was grade 2. In mixed MC-IDC, there were nests and tubules of tumor typical of NOS type invasive ductal carcinoma (IDC-NOS) in addition to mucinous component. They were graded as 1 in 1 case; grade 2 in 2 cases and 3 in 1 case. Another case was grade 3 mixed cribriform and mucinous carcinoma. Table 1 compares cytological and histopathological diagnoses. In 3 cases (case\# 3, 7, 8), FNAC diagnosis of mucinous carcinoma was confirmed by histopathological examination. However, in 2 cases (case\# 2, 5) of mucinous carcinoma diagnosed on FNAC, the histological examination revealed mixed MC-IDC. In 2 cases (case\# 4, 6) histopathology confirmed the FNAC diagnosis of mixed MC-IDC; while histopathology revealed mixed cribriform \& mucinous carcinoma in case\# 1 , which was diagnosed as mixed MC-IDC in FNAC.

\section{DISCUSSION}

Pure mucinous carcinoma is a rare histologic type of mammary neoplasm, seen in elderly women and has a better prognosis than usual invasive ductal carcinoma. ${ }^{4,10,12}$ In this study, pure mucinous carcinoma constituted $1 \%$ of breast cancer with mean age of 63 years.

The cytologic diagnosis of mucinous carcinoma is based on a combination of the characteristic morphology and abundant extracellular mucin. Aspirate usually yields visible jelly like mucin. The lake of mucin bathing bland tumor cells is a very distinctive clue to the identification of MC. This feature was seen in all cases in the present study. However, the presence of only mucin does not warrant a diagnosis of $\mathrm{MC}$ as other breast lesions i.e. mucocele like lesions, mucinous cyst and mucinous spherulosis. also show extracellular mucin. In this study, there were no cases of benign mucin producing lesion. Stanley MW et $\mathrm{al}^{13}$ mentioned that mucin is more easily identified on Romanovsky-stained smears than Papanicolaou-stained smears.

The thin wall blood vessels were seen in all cases of pure MC and 2 cases of mixed MC-IDC in this study; however, this feature was observed in approximately $50 \%$ cases of MC in other studies. ${ }^{13,14}$ This discrepancy is likely due to small number of cases in present study. Nuclear atypia in pure $\mathrm{MC}$ is minimal to mild with grade one nuclear features ${ }^{15,16}$ and we observed similar findings.

This study included 4 cases of MC-IDC. This diagnosis was made based on following cytological features: 1) one or more smears lack mucin 2) one or more smears are more cellular and nuclear atypia is much more higher than in typical pure mucinous carcinoma 3) nucleoli are unduely prominent 4) necrosis is present. In these cases, we noticed abundant extracellular mucin as well. Cytological diagnoses in these cases were confirmed by histological examination. Stanley MW et al $^{13}$ developed morphological features for

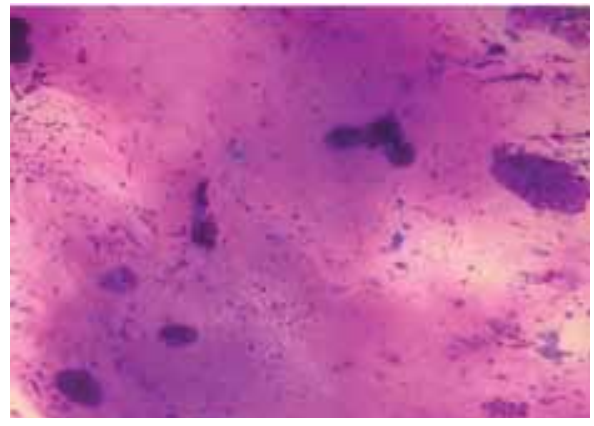

Figure 1: FNAC of pure mucinous carcinoma; Aggregates of bland cells in lakes of mucin. (Giemsa stain, X40).

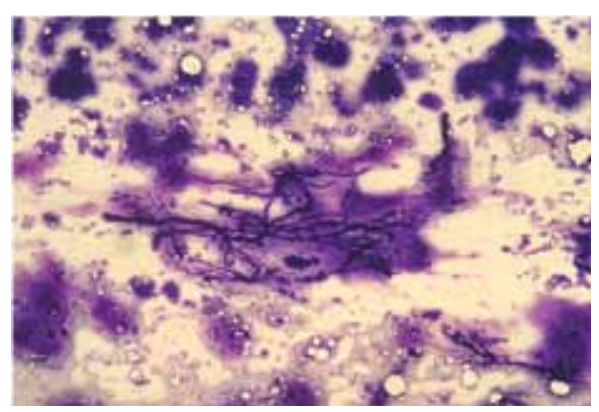

Figure 2: FNAC of pure mucinous carcinoma; Chickenwire vasculature with abundant mucin and aggregates of tumor cells on the background. (Giemsa stain, X100).

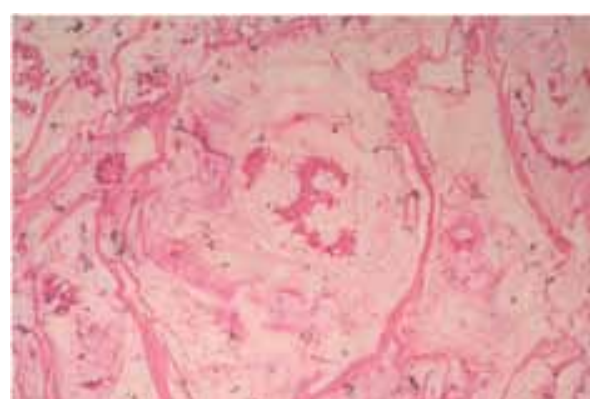

Figure 3: Pure mucinous carcinoma; Tumor cells floating in mucin, which is separated by thin fibrous septa. (HE stain, X100).

diagnosis of mixed MC-IDC, which were somewhat similar to above mentioned features. Duane et $\mathrm{al}^{3}$ diagnosed two of four mixed tumors based on the presence of large atypical nuclei. We observed large atypical nuclei in all cases of mixed tumors and none of the cases of pure mucinous carcinoma.

Semi-quantitative study of extracellular mucin and large numbers of pleomorphic cells strongly suggests a mixed MC-IDC. ${ }^{13}$ A case with abundant extracellular mucin on all smears, no pleomorphism noted after careful study, and no necrosis is probably pure MC..$^{13}$

In 1 case diagnosed as mixed MC-IDC on FNAC, the histology revealed mixed cribriform and mucinous carcinoma. This is due to rarity of cribriform carcinoma 
and lack of experience to identify cribriform carcinoma on cytologic smears. In 2 cases, the diagnosis of mucinous carcinoma was made on cytology and histopathological examination revealed mixed MC-IDC. Inadequate sampling may be the reason and IDC component was not sampled on FNA.

\section{CONCLUSION}

Though mucinous carcinoma may appear clinically and radiologically benign, FNAC plays important role in the correct pre-operative diagnosis. Significant nuclear pleomorphism and necrosis, in addition to extracellular mucin suggests mixed MC-IDC.

\section{REFERENCES}

1. Hammedi F, Trabelsi A, Abdelkrim SB, et al. Mucinous carcinoma with axillary lymphnode metastasis in a male breast: a case report. North Am J Med Sci 2010;2:111-3.

2. Suen $\mathrm{KC}$, editor. Atlas and text of aspiration biopsy cytology. Williams \& Wilkins: Baltimore;1990.pp109.

3. Duane GB, Kanter MH, Branigan T, Chang C. A morphologic and morphometric study of cells from colloid carcinoma of breast obtained by fine needle aspiration. Distinction from other breast lesions. Acta Cytol 1987;31:742-50.

4. Di Saverio S, Gutierrez J, Avisar E. A retrospective review with long term follow up of 11,400 cases of pure mucinous carcinoma. Breast Cancer Res Treat 2008;111:541-7.
5. Andre S, Cunha F, Bernardo M, Meneses e Sousa J, Cortez F, Soares J. Mucinous carcinoma of the breast: a pathologic study of 82 cases. J Surg Oncol 1995;58:162-7.

6. Diab SG, Clark GM, Osborne CK, Libby A, Allred DC, Elledge RM. Tumor characteristics and clinical outcome of tubular and mucinous breast carcinomas. J Clin Oncol 1997;17:1442-8.

7. Tellem M, Nedwich A, Amenta PS, Imbriglia JE. Mucin-producing carcinoma of the breast. Tissue culture, histochemical ands electron microscopic study. Cancer 1966;19:573-84.

8. Nagoo KS, Rohaizak M, Naqiyah I, Shahrun Niza AS. Male breast cancer: experience from a Malaysian tertiary centre. Singapore Med J 2009; 50: 519-21.

9. Fentiman IS, Fourquet A, Hotobagyi GN. Male breast cancer. Lancet 2006; 367: 595-604.

10. Peng L, Sun Q, Liang ZY, Zhou YD, Mao F, Guan JH. Pure mucinous carcinoma of the breast: a clinicopathologic analysis with 56 patients. Chin Med Sci J 2010;25:115-8.

11. Jayaram G, Alhady SF, Yip CH. Cytologic analysis of breast lesions. A review of 780 cases. Malays J Pathol 1996;18:81-7.

12. Komaki K, Sakamoto GA, Sugano H, Morimoto T, Monden Y. Mucinous carcinoma of the breast in Japan: a prognostic analysis based on morphological features. Cancer 1988;61: 989-96.

13. Stanley MW, Tani EM, Skoog L. Mucinous breast carcinoma and mixed mucinous- infiltrating ductal carcinoma: A comparative study. Diagn Cytopathol 1989;5:134-8.

14. Fanning TV, Sneige N, Staerkel G. Mucinous breast lesions: Fine needle aspiration findings. Acta Cytol 1990;34:754-5.

15. Norris HJ, Taylor HB. Prognosis of mucinous (gelatinous) carcinoma of the breast. Cancer 1965;18: 879-85. 